\title{
Early Venous Thromboembolism Is A Strong Prognostic Factor In Patients With Advanced Pancreatic Ductal Adenocarcinoma
}

\section{Mathilde Barrau}

CHU Saint-Étienne: Centre Hospitalier Universitaire de Saint-Etienne

\section{Khawla Maoui}

CHU Saint-Étienne: Centre Hospitalier Universitaire de Saint-Etienne

\section{Bertrand Le Roy}

CHU Saint-Étienne: Centre Hospitalier Universitaire de Saint-Etienne

\section{Xavier Roblin}

CHU Saint-Étienne: Centre Hospitalier Universitaire de Saint-Etienne

\section{Patrick Mismetti}

CHU Saint-Étienne: Centre Hospitalier Universitaire de Saint-Etienne

\section{Jean-Marc Phelip}

CHU Saint-Étienne: Centre Hospitalier Universitaire de Saint-Etienne

\section{Nicolas WILLIET ( $\nabla$ nwilliet@yahoo.fr)}

CHU Saint-Étienne: Centre Hospitalier Universitaire de Saint-Etienne https://orcid.org/0000-0002-72965464

\section{Original Article - Clinical Oncology}

Keywords: pancreatic cancer, venous thromboembolism, prognostic factors, antithrombotic treatment

Posted Date: February 2nd, 2021

DOI: https://doi.org/10.21203/rs.3.rs-166604/v1

License: (c) (1) This work is licensed under a Creative Commons Attribution 4.0 International License. Read Full License

Version of Record: A version of this preprint was published at Journal of Cancer Research and Clinical Oncology on March 14th, 2021. See the published version at https://doi.org/10.1007/s00432-021-03590$\mathrm{x}$. 


\section{Abstract}

\section{Background}

There is still controversial data regarding prognostic value of Venous ThromboEmbolism (VTE) in advanced Pancreatic Ductal AdenoCarcinoma (PDAC) and thromboprophylaxis is poorly prescribed despite international recommendations.

\section{Methods}

Medical charts of patients consecutively treated for advanced PDAC from 2010 to 2019 were retrospectively reviewed. Progression-free survival (PFS) and overall survival (OS) were estimated using Kaplan-Meier method. Prognostic Factors were identified using a multivariate Cox's proportional hazard model. Early VTE was defined as VTE occurring within the third months from PDAC diagnosis.

Results

A total of 174 patients were included (median age: 67 years; males: $55.2 \%$; performance status (PS) 0-1: $88.5 \%$ ) with metastatic disease in $74.7 \%$. At baseline, Khorana score was high ( $\geq 3)$ in the vast majority of cases (93.7\%). The cumulative incidences of VTE were $12.4 \%$ (95\% Cl: $7.3-17.2)$ at 3 months, $20.4 \%$ (95\% Cl: $13.9-26.4)$ at 6 months and $28.1 \%(95 \% \mathrm{Cl}$ : 20.0-35.3) at 12 months. Early VTE was associated with shorter PFS (3.8 months vs. 7.1 months; $\mathrm{HR}=2.02 ; 95 \% \mathrm{Cl}$ : 1.21-3.37; $\mathrm{p}=0.006)$ and shorter OS (8.0 months vs. 14.1 months; $\mathrm{HR}=2.42 ; 95 \% \mathrm{Cl}: 1.37-4.30 ; \mathrm{p}=0.002$ ) compared to the remnant patients, independently of the other prognostic factors including PS, liver metastases, carcinomatosis, and chemotherapy regimen.

Conclusion

Early VTE is a strong prognostic factor in advanced PDAC and occurs in about 1 in 10 patients.

\section{Introduction}

The risk of Venous ThromboEmbolism (VTE) is increased in cancer patients due to a prothrombotic and proangiogenic state involving numerous mechanisms such as tumor overexpression of tissue factor (Khorana and Fine 2004). Chemotherapy, distant metastases and the primary site of cancer are additional risk factors for VTE (Blom et al. 2006).

Pancreatic ductal adenocarcinoma (PDAC) is among the most common malignancies associated with this risk (Blom et al. 2006). In the majority of PDAC cohorts, the prevalence of VTE ranges from 10-30\% (Epstein et al. 2012; Lee et al. 2016; Frere et al. 2019). However, VTE can occur at various times of the disease. Hence, the cumulative incidence of VTE appears more informative but has been less frequently reported in the literature (Lee et al. 2016; Gade et al. 2017; Chen et al. 2018; Frere et al. 2019; Godinho et al. 2020; van Es et al. 2020). 
The Khorana score was developed and validated in 2008 (Khorana et al. 2008) as a predictive model for chemotherapy-associated thrombosis. This score takes into account the primary site of cancer, prechemotherapy platelets count, hemoglobin level, use of erythropoiesis-stimulating agents, leucocytes count, and body mass index (BMI) (Khorana et al. 2008). According to this, all patients with PDAC have a Khorana score of at least 2 points (moderate risk) and a significant proportion of them have a high risk (score $\geq 3$ ) and should undergoing primary thromboprophylaxis as recommended since 2013 with a Grade 1B evidence level.

However, the benefit of thromboprophylaxis in these patients continues to be under-recognized worldwide. Indeed, except for the perioperative setting and for hospitalized patients, ambulatory thromboprophylaxis remains underused because of high rates of VTE recurrence, and potential bleeding complications during VTE treatment (Key et al. 2020). Due to their short life expectancy, there has been no strong evidence that inhibition of the coagulation cascade improves survival in patients with advanced PDAC (Frere et al. 2019). Moreover, there are still controversial data regarding the prognostic value of VTE in PDAC (Mandalà et al. 2007; Epstein et al. 2012; Lee et al. 2016; Chen et al. 2018; Frere et al. 2019). Given VTE is a time-dependent factor there is growing evidence that early-VTE predicts survivals (Mandalà et al. 2007; Chen et al. 2018; Kim et al. 2018). Therefore this retrospective cohort study was conducted to assess the impact of early VTE on survivals.

\section{Methods}

\section{Study design and objectives}

All consecutive patients treated for PDAC from 2010 to 2019 at the University Hospital of Saint-Etienne (France) were reviewed. Until June 2018, patients were identified by using the International Classifications of Diseases (CIM10). Their data were collected from their electronic charts in agreement with the Declaration of Helsinki. The Ethics Committee of Hospital Saint Etienne approved the study protocol (IRBN632017/CHUSTE). From June 2018 to June 2019, this retrospective cohort was pooled to a prospective cohort, observational and carried-out in the same centre (IRBN362018/CHUSTE).

The primary objective of this study was to assess the prognostic value of VTE and early VTE in advanced PDAC. Secondary objective was to report the cumulative incidence of VTE in patients with advanced PDAC. Inclusion criteria were patients with advanced PDAC at baseline (locally advanced or metastatic disease) or in recurrence in case of tumor previously resected.

\section{Data collection and definitions}

Patient data collection included demographic characteristics (age, sex, body mass index [BMI]), comorbidities (diabetes, tobacco consumption), the performance status (PS) by the Eastern Cooperative Oncology Group Performance Status Scale (ECOG-PS) and nutritional status (albumin level). 
Regarding cancer disease, the following data were collected: primary tumor location, disease stage (locally advanced, metastatic or in recurrence), CA 19 - 9 at baseline, and first-line chemotherapy regimen. Baseline was defined as the time of diagnosis for advanced disease or as the time of recurrence for tumors which were previously resected.

Progression-free survival (PFS) was defined from the date of baseline to disease-progression (according to Response Evaluation Criteria in Solid Tumors (RECIST v1) criteria), or to date of death or loss to followup. Overall survival (OS) was defined from the date of baseline to death or loss to follow-up.

Regarding VTE events, the following data were collected: date of VTE occurrence, location of the thrombosis (visceral, extra-visceral), symptomatic or asymptomatic feature, treatment, duration and response to the treatment. Time-to-progression and Time-to-death were defined from the date of VTE occurrence to the date of disease progression or death, respectively, or loss to follow-up. Early VTE was defined as the occurrence of VTE within the third months from baseline.

\section{Statistical analysis}

Survivals (PFS, OS) and time from VTE to event (progression, death) were estimated using Kaplan Meier Method. Prognostic factors were identified using Cox's proportional hazards regression. Multivariate analyses were performed either by stepwise method or by selecting variables according their corresponding $\mathrm{p}$ value $(\leq \mathbf{0 . 0 5})$ in univariate analyse. For each continuous variable, cut-offs used to evaluate the prognosis were in accordance with the literature (e.g.: BMI $\geq 25$ or $30 \mathrm{~kg} / \mathrm{m}^{2}$, platelets count $\geq 350 \times 10^{9} / \mathrm{L}$, leucocytes count $\geq 11 \times 10^{9} / \mathrm{L}$, hemoglobin $<100 \mathrm{~g} / \mathrm{L}$ ). The cumulative incidence of VTE was estimated by using the inverse Kaplan Meier method.

Quantitative variables were reported as median with corresponding interquartile range and were compared by Wilcoxon test. Qualitative variables were reported as numbers and percentages and compared using chi2 or fisher test. All statistical analyses were performed using $R \circledast$ version 3.2.2 ( $R$ project, Auckland, New Zealand).

\section{Results}

\section{Whole study population}

A total of 174 patients matching with inclusion criteria were identified. Patients and disease characteristics were reported in Table 1, as well as corresponding data for the group of patients who experienced VTE during follow-up $(n=46)$ and data for those who did not $(n=128)$. Forty six patients $(26.4 \%)$ had at least one VTE event during follow-up. There was no statistically significant difference between the two groups regarding their main characteristics. The median age was 67 years [60-75] with a sex ratio close to 1 . At time of diagnosis, most patients (88.5\%) had an ECOG-PS of $0-1$, about $1 / 3$ $(32.8 \%)$ had diabetes and $37.4 \%$ had history of tobacco consumption. At baseline, median of albumin was $32.6 \mathrm{~g} / \mathrm{L}$ [27.2-37.9]. 
Table 1 Baseline characteristics of the population study

\begin{tabular}{|c|c|c|c|c|}
\hline Variables & $\begin{array}{l}\text { Population } \\
\text { Study } \\
(n=174)\end{array}$ & $\begin{array}{l}\text { No VTE } \\
\text { during follow- } \\
\text { up } \\
(n=128)\end{array}$ & $\begin{array}{l}\text { VTE } \\
\text { during follow- } \\
\text { up } \\
(n=46)\end{array}$ & $\begin{array}{l}P \\
\text { value }\end{array}$ \\
\hline Age, mean [IQR] & $67[60-75]$ & $68[61-75]$ & $65[57.5-73]$ & 0.302 \\
\hline Sex Male & $96(55.2 \%)$ & $71(55.5 \%)$ & $25(54.3 \%)$ & 1 \\
\hline History of Tobacco & $65(37.4 \%)$ & $51(39.8 \%)$ & $14(30.4 \%)$ & 0.37 \\
\hline Diabetes & $57(32.8 \%)$ & $41(32 \%)$ & $16(34.8 \%)$ & 0.734 \\
\hline $\begin{array}{l}\text { Diabetes duration (years) mean } \\
{[I Q R]}\end{array}$ & $6.4[0.5-9]$ & $6.3[0.5-10.2]$ & $7.1[2.2-7.6]$ & 0.982 \\
\hline Body mass index mean [IQR] & $24[21-27.8]$ & $24[21-27]$ & 25 [22-29] & 0.052 \\
\hline \multicolumn{5}{|l|}{ Performance status } \\
\hline ECOG-O & $61(35.1 \%)$ & $42(32.8 \%)$ & $19(41.3 \%)$ & 0.397 \\
\hline ECOG-1 & $93(53.4 \%)$ & $71(55.5 \%)$ & $22(47.8 \%)$ & \\
\hline ECOG-2 & $20(11.5 \%)$ & $15(11.7 \%)$ & $5(10.9 \%)$ & \\
\hline CA19.9_C1 & $\begin{array}{l}353.5[87.5- \\
2761]\end{array}$ & 283 [65-1329] & $\begin{array}{l}648[184.5- \\
4576]\end{array}$ & 0.133 \\
\hline missing data & $44(25.3 \%)$ & $34(26.6 \%)$ & $10(21.7 \%)$ & - \\
\hline CRP.a.C1 & $17[7-43.5]$ & $16.5[7-40.8]$ & $19.5[9-51.5]$ & 0.364 \\
\hline missing data & $28(16.1 \%)$ & $22(17.2 \%)$ & $6(13 \%)$ & - \\
\hline AlbumineC1 & $32.6[27.2-37.9]$ & $33[29-37]$ & $32[25-38]$ & 0.766 \\
\hline Missing data & $52(29.9 \%)$ & $43(33.6 \%)$ & $9(19.6 \%)$ & - \\
\hline Leucocytes count $\geq 11 \times 10^{9} / \mathrm{L}$ & $34(21.4 \%)$ & $21(18.3 \%)$ & $13(29.5 \%)$ & 0.18 \\
\hline Hemoglobin $\leq 100 \mathrm{~g} / \mathrm{L}$ & $19(11.9 \%)$ & $10(8.7 \%)$ & $9(20.5 \%)$ & 0.08 \\
\hline Platelets count $\geq 350 \times 10^{9} / \mathrm{L}$ & $25(15.7 \%)$ & $18(15.7 \%)$ & $7(15.9 \%)$ & 1 \\
\hline Khorana Score & $3[3-4]$ & $3[3-3]$ & $3[3-4]$ & 0.114 \\
\hline$\geq 3$ & 149 ( $93.7 \%)$ & $108(93.9 \%)$ & 41 ( $93.2 \%)$ & 1 \\
\hline$\geq 4$ & 44 ( $27.7 \%)$ & $27(23.5 \%)$ & 17 ( $38.6 \%)$ & 0.09 \\
\hline missing data & $15(8.6 \%)$ & $13(10.2 \%)$ & $2(4.3 \%)$ & - \\
\hline
\end{tabular}




\begin{tabular}{|c|c|c|c|c|}
\hline Variables & $\begin{array}{l}\text { Population } \\
\text { Study } \\
(n=174)\end{array}$ & $\begin{array}{l}\text { No VTE } \\
\text { during follow- } \\
\text { up } \\
(n=128)\end{array}$ & $\begin{array}{l}\text { VTE } \\
\text { during follow- } \\
\text { up } \\
(n=46)\end{array}$ & $\begin{array}{l}P \\
\text { value }\end{array}$ \\
\hline \multicolumn{5}{|l|}{ Disease stage } \\
\hline Non-metastatic & 44 ( $25.3 \%)$ & $31(24.2 \%)$ & $13(28.3 \%)$ & 0.59 \\
\hline Metastatic & 130 ( $74.7 \%)$ & $97(75.8 \%)$ & 33 ( $71.7 \%)$ & 0.59 \\
\hline Prior surgery & 30 ( $17.2 \%)$ & $24(18.8 \%)$ & $6(13 \%)$ & 0.50 \\
\hline \multicolumn{5}{|c|}{ Location of primary tumor } \\
\hline Head & $93(53.4 \%)$ & $73(57 \%)$ & $20(43.5 \%)$ & 0.634 \\
\hline Body & $29(16.7 \%)$ & $19(14.8 \%)$ & $10(21.7 \%)$ & \\
\hline Tail & $27(15.5 \%)$ & $21(16.4 \%)$ & $6(13 \%)$ & \\
\hline missing data & $25(14.4 \%)$ & $15(11.7 \%)$ & $10(21.7 \%)$ & \\
\hline \multicolumn{5}{|l|}{ Metastases sites } \\
\hline Liver & 78 ( $44.8 \%)$ & $60(46.9 \%)$ & $18(39.1 \%)$ & 0.366 \\
\hline Lung & $20(11.5 \%)$ & $16(12.5 \%)$ & $4(8.7 \%)$ & 0.489 \\
\hline Bone & $6(3.4 \%)$ & $6(4.7 \%)$ & $0(0 \%)$ & 0.34 \\
\hline Carcinomatosis & $33(19.1 \%)$ & $22(17.3 \%)$ & $11(23.9 \%)$ & 0.45 \\
\hline Number of sites & $1[0-1]$ & $1[0-1]$ & $1[0-1]$ & 0.603 \\
\hline \multicolumn{5}{|c|}{ Chemotherapy regimen } \\
\hline 5FU-based & $92(52.9 \%)$ & $68(53.1 \%)$ & $24(52.2 \%)$ & 1 \\
\hline Gemcitabine-based & $82(47.1 \%)$ & $60(46.9 \%)$ & $22(47.8 \%)$ & \\
\hline Intensive & 106 ( $60.9 \%)$ & 78 ( $60.9 \%)$ & $28(60.9 \%)$ & 0.87 \\
\hline Doublet 5FU-based & $12(6.9 \%)$ & $8(6.2 \%)$ & $4(8.7 \%)$ & \\
\hline Gemcitabine only & $56(32.2 \%)$ & $42(32.8 \%)$ & $14(30.4 \%)$ & \\
\hline
\end{tabular}

About half of patients had a primary tumor located in the head of the pancreas and metastatic disease in $74.7 \%$ (17.2\% after prior surgery of the primitive tumor). At baseline, median CA19.9 was $353.5 \mathrm{UI} / \mathrm{L}$ [87.5-2761], and $17 \mathrm{mg} / \mathrm{L}$ [7.0-43.5] for CRP. About 2/3 of patients received intensive chemotherapy (FOLFIRINOX or Gemcitabine/Nab-Paclitaxel). About 1/3 were treated with Gemcitabine only. The 
Khorana score at baseline was similar between patients who experience VTE vs. those who did not (median: 3 [3-4]) despite a statistical trend for patients with score $\geq 4$ in VTE group (38.6\% vs. $23.5 \% ; p=$ $0.11)$.

\section{Incidence of Venous ThromboEmbolism}

During a median follow-up of 9.3 months [5.1-16.7], 46 patients (26.4\%) experienced VTE. In the whole study population, the cumulative incidence of VTE was $12.4 \%$ (95\% Cl: $7.3-17.2)$ at 3 months, $20.4 \%$ (95\% Cl: $13.9-26.4)$ at 6 months, and $28.1 \%$ (95\% Cl: 20.0-35.3) at 12 months (Fig. 1A). Patients with Khorana score $\geq 4$ had a doubled risk of VTE $(H R=1.96 ; 95 \% \mathrm{Cl}: 1.05-3.65$; Log-rank $p=0.03)$ with the following corresponding cumulative incidences: $21.0 \%(95 \% \mathrm{Cl}: 7.8-32.4)$ at 3 months, $36.3 \%(95 \% \mathrm{Cl}$ : 18.6-50.2) at 6 months and $40.9 \%$ (95\%Cl: 21.3-55.5) at 12 months (Fig. 1B).

Among patients who experienced VTE, $15.2 \%$ (95\% Cl: 4.2-25) had it at baseline, 45.7\% (95\% Cl: 29.2$58.3)$ within three months and more than 2/3 (71.7\%; 95\%Cl: 55.2-82.2) within six months.

\section{Clinical presentation and treatment of venous thromboembolism events}

Of the 46 patients who experienced VTE during follow-up, the majority of VTE was symptomatic (78.3\%) and extra-abdominal (76.1\%) including pulmonary embolism in 30.4\%. Most of them were treated with Low Molecular Weight Heparins (LMWHs) (69.6\%). Vitamin K antagonists (VKA) (4.3\%), direct oral anticoagulants (DOAC) $(2.1 \%)$, and unfractionated heparin $(2.1 \%)$ were rarely used and $17.4 \%$ of patients did not received specific treatment for VTE. One patient (2.1\%) underwent vena cava filter. Under anticoagulant agents, VTE was considered as progressive (locally, or by the occurrence of a second TVE site), stable or reduced in $17.4 \%, 67.4 \%$ and $13 \%$, respectively. Second-line anticoagulation strategy was dominated by an increase of LMWH dose $(n=4)$, or maintenance the same strategy $(n=4)$. Bleeding complication was observed in $15.2 \%$ with 1 related death $(2.2 \%)$ by digestive hemorrhage.

\section{Survivals}

In the whole study population the median PFS was 9.3 months (95\% Cl: $5.1-16.7)$. In univariate analysis, VTE occurrence during follow-up was not predictive of progression $(p=0.18)$. In contrast, there was a correlation between time from cancer diagnosis to VTE and PFS $(p=0.006 ; H R=0.89)$. Patients who experienced early VTE (within three months) were associated with shorter PFS than the others: 3.8 months (95\%Cl: $2.7-10.1)$ vs. 7.1 months (95\%Cl: $6.2-8.4)$, respectively ( $\mathrm{HR}=2.02 ; 95 \% \mathrm{Cl}: 1.21-3.37 ; \mathrm{p}$ $=0.006)($ Fig. 2A). After multivariate analysis (Table 2), early VTE remained predictor of PFS, independently to other prognostic factors such as ECOG-PS $>1(p<0.01 ; \mathrm{HR}=2.60 ; 95 \% \mathrm{Cl}$ : 1.49-4.52), $\mathrm{BMI} \geq 25$ ( $p=0.002 ; \mathrm{HR}=0.57 ; 95 \% \mathrm{Cl}: 0.4-0.82)$, liver metastasis $(p=0.003 ; \mathrm{HR}=1.71 ; 95 \% \mathrm{Cl}: 1.20-$ 2.45), carcinomatosis ( $p=0.03 ; \mathrm{HR}=1.60 ; 95 \% \mathrm{Cl}: 1.04-2.46)$, and chemotherapy regimen (Gemcitabinebased vs. fluoropyrimidine-based: $p=0.06 ; \mathrm{HR}=1.45 ; 95 \% \mathrm{Cl}$ : 0.98-2.15). 
Table 2 Prognostic factors for progression-free survival

\begin{tabular}{|c|c|c|c|c|}
\hline & \multicolumn{2}{|c|}{ Univariate analyses } & \multicolumn{2}{|c|}{ Multivariate analyses } \\
\hline & $\begin{array}{l}\mathrm{p} \\
\text { value }\end{array}$ & $\mathrm{HR}[95 \% \mathrm{Cl}]$ & $\begin{array}{l}\mathrm{p} \\
\text { value }\end{array}$ & $\mathrm{HR}[95 \% \mathrm{Cl}]$ \\
\hline Age & 0.12 & $1.01[1-1.03]$ & & \\
\hline Age $>75$ years & 0.11 & $\begin{array}{l}1.37[0.93- \\
2.03]\end{array}$ & 0.69 & $\begin{array}{l}0.91[0.58- \\
1.43]^{[}\end{array}$ \\
\hline Sex: Male vs Female & 0.65 & $\begin{array}{l}0.93[0.67- \\
1.28]\end{array}$ & & \\
\hline Tobacco vs No Tobacco & 0.24 & $1.22[0.87-1.7]$ & & \\
\hline Diabetes vs No Diabetes & 0.79 & $\begin{array}{l}1.05[0.74- \\
1.49]\end{array}$ & & \\
\hline Body mass index (BMI) & 0.09 & $\begin{array}{l}0.97[0.93- \\
1.01]\end{array}$ & & \\
\hline $\mathrm{BMI} \geq 25$ & 0.01 & $0.64[0.46-0.9]$ & 0.002 & $0.57[0.4-0.82]$ \\
\hline Performance Status: ECOG 2 vs $0-1$ & $<.001$ & $\begin{array}{l}2.78[1.69- \\
4.57]\end{array}$ & $<.001$ & $2.6[1.49-4.52]$ \\
\hline $\begin{array}{l}\text { Time from cancer diagnostic to VTE } \\
\text { (months) }\end{array}$ & 0.006 & $\begin{array}{l}0.89[0.86- \\
0.91]\end{array}$ & & \\
\hline Early VTE vs No Early VTE & 0.01 & $\begin{array}{l}2.02[1.21- \\
3.37]\end{array}$ & 0.037 & $1.8[1.04-3.12]$ \\
\hline Stage : Metastatic vs Locally Advanced & 0.06 & $\begin{array}{l}1.42[0.98- \\
2.06]\end{array}$ & & \\
\hline No prior surgery vs prior surgery & 0.24 & $\begin{array}{l}1.33[0.83- \\
2.14]\end{array}$ & & \\
\hline \multicolumn{5}{|l|}{ Primitive Tumor location } \\
\hline head vs body & 0.6 & $\begin{array}{l}0.89[0.57- \\
1.39]\end{array}$ & & \\
\hline tail vs body & 0.37 & $\begin{array}{l}1.28[0.74- \\
2.21]\end{array}$ & & \\
\hline Liver metastases & 0.035 & $\begin{array}{l}1.42[1.03- \\
1.97]\end{array}$ & 0.003 & $1.71[1.2-2.45]$ \\
\hline Lung metastases & 0.91 & $\begin{array}{l}1.03[0.62- \\
1.71]\end{array}$ & & \\
\hline Bone metastases & 0.67 & $\begin{array}{l}0.82[0.34- \\
2.01]\end{array}$ & & \\
\hline
\end{tabular}




\begin{tabular}{|c|c|c|c|c|}
\hline \multirow[b]{2}{*}{ Carcinomatosis } & \multicolumn{2}{|c|}{ Univariate analyses } & \multicolumn{2}{|c|}{ Multivariate analyses } \\
\hline & 0.005 & $\begin{array}{l}1.77[1.18- \\
2.64]\end{array}$ & 0.032 & $1.6[1.04-2.46]$ \\
\hline $\begin{array}{l}\text { Gemcitabine-based vs 5FU-based CT } \\
\text { regimen }\end{array}$ & 0.012 & $1.53[1.1-2.12]$ & 0.06 & $\begin{array}{l}1.45[0.98- \\
2.15]\end{array}$ \\
\hline $\begin{array}{l}\text { Doublet chemotherapy vs intensive } \\
\text { CT regimen }\end{array}$ & 0.73 & $1.12[0.6-2.1]$ & & \\
\hline Gemcitabine alone vs intensive CT regimen & 0.015 & $\begin{array}{l}1.55[1.09- \\
2.21]\end{array}$ & & \\
\hline
\end{tabular}

In the whole study population the median OS was 13.3 months (95\%Cl: 10.6-16.7). In univariate analysis, VTE occurrence during follow-up was not predictive of death $(p=0.97)$. In contrast, there was a correlation between time from cancer diagnosis to VTE and OS ( $<<0.001 ; H R=0.88)$. Patients who experienced early VTE were associated with shorter OS than the others: 8.0 months ( $95 \% \mathrm{Cl}$ : $4.7-\mathrm{NA})$ vs. 14.1 months $(95 \% \mathrm{Cl}: 11.1-17.4)$ respectively $(\mathrm{HR}=2.42 ; 95 \% \mathrm{Cl}: 1.37-4.30 ; \mathrm{p}=0.002)$ (Fig. 2B). The other prognostic factors for OS were reported in Table 3. Early VTE remained predictor of OS $(p=0.02 ; H R=$ $2.05)$ independently to PS-2 vs PS-1-2 ( $<<0.01 ; H R=2.43)$, liver metastasis $(p=0.02 ; H R=1.64)$, carcinomatosis $(p=0.05 ; H R=1.64)$, chemotherapy regimen $(p=0.18)$ and age $>75$ years $(p=0.6)$. 
Table 3 Prognostic factors for overall survival

\begin{tabular}{|c|c|c|c|c|}
\hline & \multicolumn{2}{|c|}{ Univariate analyses } & \multicolumn{2}{|c|}{ Multivariate analyses } \\
\hline & $p$ value & $\mathrm{HR}[95 \% \mathrm{Cl}]$ & $\begin{array}{l}\mathrm{p} \\
\text { value }\end{array}$ & $\mathrm{HR}[95 \% \mathrm{Cl}]$ \\
\hline Age & 0.17 & $\begin{array}{l}1.02[0.99- \\
1.04]\end{array}$ & & \\
\hline Age $>75$ years & 0.02 & $\begin{array}{l}1.66[1.07- \\
2.59]\end{array}$ & 0.6 & $\begin{array}{l}1.15[0.69- \\
1.91]\end{array}$ \\
\hline Sex: Male vs Female & 0.3 & $\begin{array}{l}0.82[0.56- \\
1.19]\end{array}$ & & \\
\hline Tobacco vs No Tobacco & 0.64 & $1.1[0.75-1.61]$ & & \\
\hline Diabetes vs No Diabetes & 0.69 & $\begin{array}{l}0.92[0.61- \\
1.39]\end{array}$ & & \\
\hline Body mass index (BMI) & 0.23 & $\begin{array}{l}0.97[0.93- \\
1.02]\end{array}$ & & \\
\hline $\mathrm{BMI} \geq 25$ & 0.3 & $0.82[0.56-1.2]$ & & \\
\hline Performance Status: ECOG 2 vs $0-1$ & $<0.001$ & $\begin{array}{l}2.66[1.55- \\
4.56]\end{array}$ & 0.004 & $2.43[1.34-4.4]$ \\
\hline $\begin{array}{l}\text { Time from cancer diagnostic to VTE } \\
\text { (months) }\end{array}$ & $<.0001$ & $0.88[0.86-0.9]$ & & \\
\hline Early VTE vs No Early VTE & $<0.001$ & $2.42[1.37-4.3]$ & 0.023 & $2.05[1.1-3.82]$ \\
\hline Stage : Metastatic vs Locally Advanced & 0.24 & $\begin{array}{l}1.29[0.84- \\
1.96]^{[}\end{array}$ & & \\
\hline No prior surgery vs prior surgery & 0.5 & $\begin{array}{l}0.85[0.52- \\
1.38]\end{array}$ & & \\
\hline \multicolumn{5}{|l|}{ Primitive Tumor location } \\
\hline head vs body & 0.52 & $\begin{array}{l}1.21[0.68- \\
2.16]\end{array}$ & & \\
\hline tail vs body & 0.22 & $\begin{array}{l}1.54[0.78- \\
3.06]\end{array}$ & & \\
\hline Liver metastases & 0.17 & $\begin{array}{l}1.31[0.89- \\
1.93]^{-}\end{array}$ & 0.023 & $\begin{array}{l}1.64[1.07- \\
2.52]\end{array}$ \\
\hline Lung metastases & 0.59 & $\begin{array}{l}0.85[0.47- \\
1.55]\end{array}$ & & \\
\hline Bone metastases & 0.33 & $\begin{array}{l}0.61[0.22- \\
1.65]\end{array}$ & & \\
\hline
\end{tabular}




\begin{tabular}{|c|c|c|c|c|}
\hline \multirow[b]{2}{*}{ Carcinomatosis } & \multicolumn{2}{|c|}{ Univariate analyses } & \multicolumn{2}{|c|}{ Multivariate analyses } \\
\hline & 0.07 & $\begin{array}{l}1.55[0.96- \\
2.48]\end{array}$ & 0.05 & $1.64[1-2.71]$ \\
\hline $\begin{array}{l}\text { Gemcitabine-based vs 5FU-based CT } \\
\text { regimen }\end{array}$ & 0.01 & $\begin{array}{l}1.65[1.13- \\
2.41]\end{array}$ & 0.18 & $\begin{array}{l}1.37[0.86- \\
2.18]\end{array}$ \\
\hline $\begin{array}{l}\text { Doublet chemotherapy vs intensive } \\
\text { CT regimen }\end{array}$ & 0.1 & $1.72[0.9-3.28]$ & & \\
\hline $\begin{array}{l}\text { Gemcitabine alone vs intensive CT } \\
\text { regimen }\end{array}$ & 0.004 & $\begin{array}{l}1.83[1.22- \\
2.76]\end{array}$ & & \\
\hline \multicolumn{5}{|c|}{$\begin{array}{l}\text { Notes. CT : chemotherapy ; ECOG: Eastern Cooperative Oncology Group; } 5 F U \text { : fluoropyrimidine; ; } \\
\text { Intensive CT : Folfirinox or Gemcitabine/Nab-Paclitaxel; VTE: Venous thromboembolism }\end{array}$} \\
\hline
\end{tabular}

The median time from VTE to progression was 1.6 months (95\%Cl: 0.0-2.8) (Supplementary Fig. 1), which means that half of patients who had VTE experienced disease progression at the same time. Half of the remnant patients had disease progression about 3 months after the occurrence of VTE.

The median time from VTE to death was 5.8 months (95\%Cl: 2.9-8.4) (Supplementary Fig. 2), but there was any difference between patients with abdominal thrombosis $(n=11)$ compared to those who had extra-abdominal thrombosis $(n=35 ; p=0.01)$, or regarding the symptomatic feature of the VTE event $(p=$ $0.85)$.

\section{Discussion}

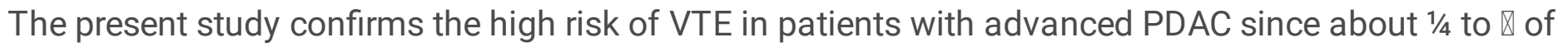
them experienced such event during follow-up. Other large cohorts had previously reported such outcomes (Lee et al. 2016; Gade et al. 2017; Chen et al. 2018; Frere et al. 2019). Thereby, the interest of thromboprophylaxis is revived for all patients with advanced PDAC as recommended since 2013 (Farge et al. 2013; Frere et al. 2019; Key et al. 2020).

However, VTE is a time dependent factor and only early VTE, defined by the occurrence of VTE within the three months from advanced PDAC diagnosis, seems prognostic in this study which involves about 1 in 10 patients. Most studies have reported VTE without taking into account its time dependent nature (Oh et al. 2008; Shaib et al. 2010; Kruger et al. 2017; Godinho et al. 2020). Only few recent studies evaluated VTE either by considering VTE as time-dependent factor in Cox model (Frere et al. 2019), or by evaluating the binary factor early VTE as this one (Mandalà et al. 2007; Chen et al. 2018; Kim et al. 2018). 
This study is one of rare that reported time from VTE to disease progression or death. Hence we showed that VTE seems in relation with tumor aggressiveness (median time to progression: 1.6 months; median time to death: 5.8 months) which raises the question about impact of anticoagulation therapy on survival. Moreover, $17.4 \%$ of patients treated for VTE experienced an extension of their thrombosis under anticoagulation and $67.4 \%$ had stability in the present study. Seven patients $(15.2 \%)$ had bleeding complication including one (2.2\%) death by digestive hemorrhage. There has been no strong evidence that thromboprophylaxis improves survival outcomes in advanced PDAC. In vitro, anticoagulation agents might have an anti-tumor effect by inhibiting metastatic spreading process. Most of randomized clinical trials that evaluated impact of anticoagulant agents on survival included all kind of primary cancers (Kakkar et al. 2004; Klerk et al. 2005). Conclusions were controversial regarding the role of anticoagulation in the improvement of survivals, and outcomes in subgroup of patients with PDAC should be interpreted with cautious (Frere et al. 2019). Corresponding data with DOAC are very scarce (Frere et al. 2019). Overall, anticoagulant use is recommended to reduce the risk of VTE (Maraveyas et al. 2012; Pelzer et al. 2015; Frere et al. 2019; Key et al. 2020) but not to improve survival in patients with cancer without VTE (van Doormaal et al. 2011). For this last reason, thromboprophylaxis is rarely prescribed for ambulatory patients in our center.

The present study is not designed to assess impact of thromboprophylaxis on survival but underlines the importance to identify patients who will experience early VTE. By definition, all patients with pancreatic cancer have a Khorana score at least 2 and the vast majority of them have a score at 3 such in this cohort. Hence, the Khorana score does not allow the identification of patients who will truly experience early VTE. Thereby, if primary thrombophylaxis is done, most of patients are overtreated with potential risk of anticoagulation-related complication. Other score should be developed to improve the identification of these patients or those who did not experience VTE within the three following months.

The ONKOTEV score is another emerging reference to predict VTE (Godinho et al. 2020). To calculate the ONKOTEV score, vascular/lymphatic compression and previous history of VTE must be known. Hence, this score is not used in clinical practice due to its complexity, which supports the interest of the development of new score more feasible and reproducible.

In conclusion, VTE occurred in about $1 / 4$ to $1 / 3$ of patients with advanced PDAC. Early VTE, defined by VTE occurrence within three months from advanced PDAC diagnostic, appears prognostic in this study which supports guidelines. However, failing to apply recommendations for primary thromboprophylaxis, further score should be developed to identify better patients who are at very high-risk for early VTE, or those who will not experience such event. Such score could avoid overtreatment of the majority of patients $(9 / 10)$.

\section{Abbreviations}




\begin{tabular}{ll} 
BMI & Body mass index \\
\hline CI & Confidence interval \\
\hline CRP & C-Reactive protein \\
\hline DOAC & Directoral anticoagulant \\
\hline ECOG & Eastern Cooperative Oncology Group \\
\hline LMWHs & Low Molecular Weight Heparins \\
\hline NPV & Negative predictive value \\
\hline OS & Overall survival \\
\hline PDAC & Pancreatic ductal adenocarcinoma \\
\hline PFS & Progression-free survival \\
\hline PPV & Positive predictive value \\
\hline PS & Performance status \\
\hline RECIST & Response Evaluation Criteria in Solid Tumors \\
\hline ROC & Receiver operating characteristic \\
\hline Se & Sensibility \\
\hline Sp & Specificity \\
\hline VTE & Venous thromboembolism
\end{tabular}

\section{Declarations}

Funding: None

Conflicts of interest: None

Data availability statement: Research data are available from Dr Nicolas Williet

Code availability: please contact Dr Nicolas Williet

Authors' contributions. MB: data collection, drafting the manuscript; KM/MF: data collection; BLR/XR/PM/JMP: interpretation of data and critical review of the manuscript; NW: conception of the study, study supervision, statistical analyses, interpretation of data, drafting the manuscript;

Ethic approval: IRBN632017/CHUSTE \& IRBN362018/CHUSTE

Consent to participate: patients deaths but they were informed that such retrospective studies are frequently performed in our institution 
Consent for publication: idem. And I declare that all co-authors are consent for the publication of this article

Acknowledgements: None

\section{References}

Blom JW, Vanderschoot JPM, Oostindiër MJ, et al (2006) Incidence of venous thrombosis in a large cohort of 66,329 cancer patients: results of a record linkage study. J Thromb Haemost JTH 4:529-535. https://doi.org/10.1111/j.1538-7836.2006.01804.x

Chen J-S, Hung C-Y, Chang H, et al (2018) Venous Thromboembolism in Asian Patients with Pancreatic Cancer Following Palliative Chemotherapy: Low Incidence but a Negative Prognosticator for Those with Early Onset. Cancers 10:. https://doi.org/10.3390/cancers10120501

Epstein AS, Soff GA, Capanu M, et al (2012) Analysis of incidence and clinical outcomes in patients with thromboembolic events and invasive exocrine pancreatic cancer. Cancer 118:3053-3061.

https://doi.org/10.1002/cncr.26600

Farge D, Debourdeau P, Beckers M, et al (2013) International clinical practice guidelines for the treatment and prophylaxis of venous thromboembolism in patients with cancer. J Thromb Haemost 11:56-70. https://doi.org/10.1111/jth.12070

Frere C, Bournet B, Gourgou S, et al (2019) Incidence of Venous Thromboembolism in Patients with Newly Diagnosed Pancreatic Cancer and Factors Associated With Outcomes. Gastroenterology. https://doi.org/10.1053/j.gastro.2019.12.009

Gade IL, Braekkan SK, Naess IA, et al (2017) The impact of initial cancer stage on the incidence of venous thromboembolism: the Scandinavian Thrombosis and Cancer (STAC) Cohort. J Thromb Haemost JTH 15:1567-1575. https://doi.org/10.1111/jth.13752

Godinho J, Casa-Nova M, Moreira-Pinto J, et al (2020) ONKOTEV Score as a Predictive Tool for Thromboembolic Events in Pancreatic Cancer-A Retrospective Analysis. The Oncologist 25:e284-e290. https://doi.org/10.1634/theoncologist.2019-0510

Kakkar AK, Levine MN, Kadziola Z, et al (2004) Low molecular weight heparin, therapy with dalteparin, and survival in advanced cancer: the fragmin advanced malignancy outcome study (FAMOUS). J Clin Oncol Off J Am Soc Clin Oncol 22:1944-1948. https://doi.org/10.1200/JC0.2004.10.002

Key NS, Khorana AA, Kuderer NM, et al (2020) Venous Thromboembolism Prophylaxis and Treatment in Patients With Cancer: ASCO Clinical Practice Guideline Update. J Clin Oncol 38:496-520. https://doi.org/10.1200/JCO.19.01461 
Khorana AA, Fine RL (2004) Pancreatic cancer and thromboembolic disease. Lancet Oncol 5:655-663. https://doi.org/10.1016/S1470-2045(04)01606-7

Khorana AA, Kuderer NM, Culakova E, et al (2008) Development and validation of a predictive model for chemotherapy-associated thrombosis. Blood 111:4902-4907. https://doi.org/10.1182/blood-2007-10116327

Kim JS, Kang EJ, Kim DS, et al (2018) Early venous thromboembolism at the beginning of palliative chemotherapy is a poor prognostic factor in patients with metastatic pancreatic cancer: a retrospective study. BMC Cancer 18:1260. https://doi.org/10.1186/s12885-018-5154-3

Klerk CPW, Smorenburg SM, Otten H-M, et al (2005) The effect of low molecular weight heparin on survival in patients with advanced malignancy. J Clin Oncol Off J Am Soc Clin Oncol 23:2130-2135. https://doi.org/10.1200/JC0.2005.03.134

Kruger S, Haas M, Burkl C, et al (2017) Incidence, outcome and risk stratification tools for venous thromboembolism in advanced pancreatic cancer - A retrospective cohort study. Thromb Res 157:9-15. https://doi.org/10.1016/j.thromres.2017.06.021

Lee J-C, Ro YS, Cho J, et al (2016) Characteristics of Venous Thromboembolism in Pancreatic Adenocarcinoma in East Asian Ethnics: A Large Population-Based Observational Study. Medicine (Baltimore) 95:e3472. https://doi.org/10.1097/MD.0000000000003472

Mandalà M, Reni M, Cascinu S, et al (2007) Venous thromboembolism predicts poor prognosis in irresectable pancreatic cancer patients. Ann Oncol 18:1660-1665.

https://doi.org/10.1093/annonc/mdm284

Maraveyas A, Waters J, Roy R, et al (2012) Gemcitabine versus gemcitabine plus dalteparin thromboprophylaxis in pancreatic cancer. Eur J Cancer Oxf Engl 1990 48:1283-1292.

https://doi.org/10.1016/j.ejca.2011.10.017

Oh SY, Kim JH, Lee K-W, et al (2008) Venous thromboembolism in patients with pancreatic adenocarcinoma: lower incidence in Asian ethnicity. Thromb Res 122:485-490.

https://doi.org/10.1016/j.thromres.2007.12.015

Pelzer U, Opitz B, Deutschinoff G, et al (2015) Efficacy of Prophylactic Low-Molecular Weight Heparin for Ambulatory Patients With Advanced Pancreatic Cancer: Outcomes From the CONKO-004 Trial. J Clin Oncol Off J Am Soc Clin Oncol 33:2028-2034. https://doi.org/10.1200/JC0.2014.55.1481

Shaib W, Deng Y, Zilterman D, et al (2010) Assessing risk and mortality of venous thromboembolism in pancreatic cancer patients. Anticancer Res 30:4261-4264

van Doormaal FF, Di Nisio M, Otten H-M, et al (2011) Randomized trial of the effect of the low molecular weight heparin nadroparin on survival in patients with cancer. J Clin Oncol Off J Am Soc Clin Oncol 
van Es N, Ventresca M, Di Nisio M, et al (2020) The Khorana score for prediction of venous thromboembolism in cancer patients: an individual patient data meta-analysis. J Thromb Haemost JTH. https://doi.org/10.1111/jth.14824

\section{Supplemental Data}

Supplementary Figures 1 and 2 not available with this version

\section{Figures}
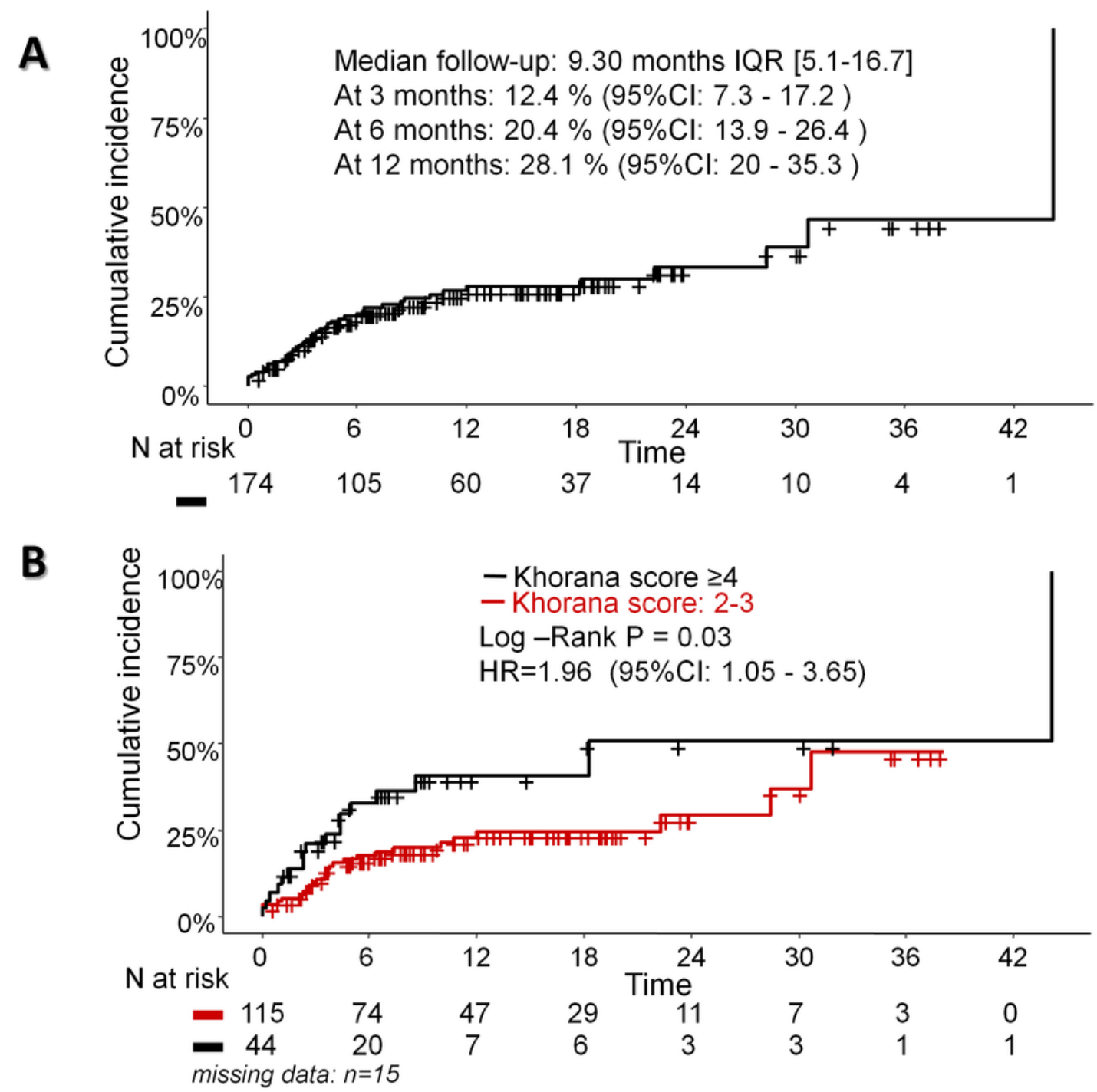

\section{Figure 1}

Cumulative incidence of venous thromboembolism A) in the whole population study B) according to a high Khorana score. 


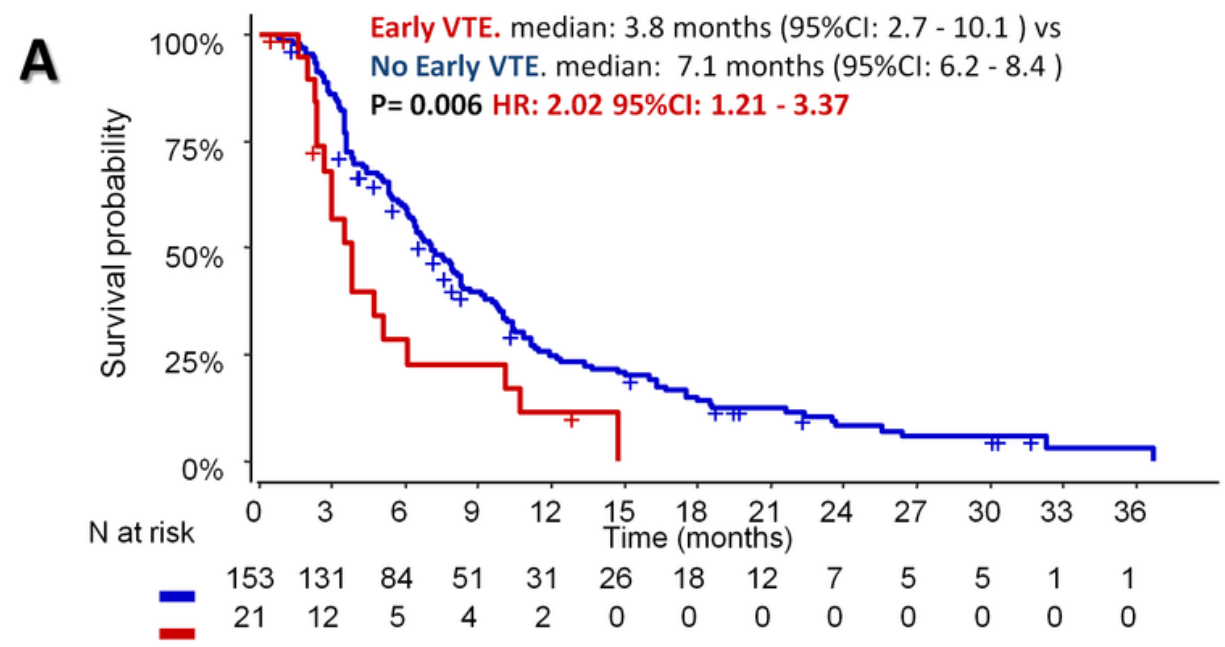

B

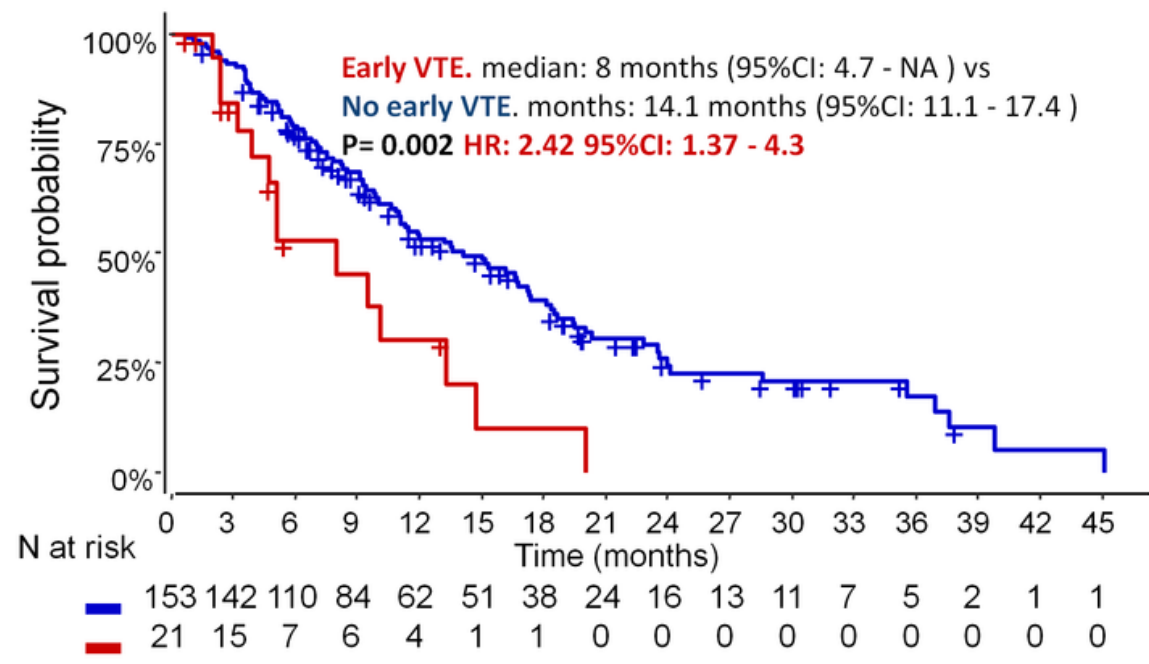

Notes. VTE: venous thromboembolism

\section{Figure 2}

Impact of early venous thromboembolism on A) Progression-free survival B) overall survival. 